\title{
Herramientas de
}

comunicación y presentación de contenidos en pregrados virtuales colombianos

Communication Tools and Content Presentation in Colombian Online Undergraduate Courses

Outils de communication et présentation de contenus chez les licences virtuelles colombiennes

Ferramentas de comunicação e apresentação de conteúdos

em cursos de graduação virtuais colombianos

Fecha de recepción: 11 DE DICIEMBRE DE 2013/Fecha de aceptación: 9 DE NOVIEMBRE DE 2015/Fecha de disponibilidad en línea: 15 DE MARZO DE 2016 Encuentre este artículo en http://magisinvestigacioneducacion.javeriana.edu.co/

\section{Resumen}

Este artículo de investigación expone los resultados de un estudio mixto que examinó las experiencias y percepciones de un grupo de profesores, coordinadores y estudiantes de programas de pregrado en modalidad virtual en Colombia acerca del empleo de herramientas para la comunicación, la presentación y la apropiación de contenidos. A pesar de algunos logros, los hallazgos evidencian la necesidad de enfatizar la interacción con carácter dialógico y la colaboración en los espacios diseñados para la comunicación. Así mismo, la representación de información requiere apelar a formas distintas al texto escrito que motiven a los estudiantes a interactuar con los materiales y estimulen su comprensión de contenidos.

\section{Palabras claves}

Educación a distancia; aprendizaje virtual; educación superior; comunicación; tecnología de la información
Geraint Paul Rees Universitat Pompeu Fabra BARCELONA, España geraintpaul.rees@upf.edu

\section{Transferencia a la práctica}

Los avances tecnológicos de la web posibilitan el empleo pedagógico de múltiples herramientas para apoyar los procesos educativos de un número cada vez más grande de estudiantes en programas de pregrado en modalidad virtual. Los resultados de esta investigación invitan a incursionar en el empleo de redes sociales, espacios de creación colectiva y espacios para el estímulo de la creatividad en aras de enriquecer la presentación y apropiación de contenidos de los programas y generar procesos de comunicación entre estudiantes y profesores más significativos y enriquecedores.

Para citar este artículo / To cite this article / Pour citer cet article / Para citar este artigo

Otero de Suárez, M. P.; Pineda-Báez, C. \& Rees, G. P. (2016). Herramientas de comunicación y presentación de contenidos en pregrados virtuales colombianos. magis, Revista Internacional de Investigación en Educación, 8 (17), 65-84. http://dx.doi.org/10.11144/Javeriana.m8-17.hcpc 


\section{Keywords}

Long distance education; online learning; higher education; communication; information technology

\section{Mots clés}

Education à distance; apprentissage virtuel; éducation supérieure; communication; technologie de l'information

\section{Abstract}

This paper presents the results of a mixed study that examined the experiences and perceptions of a group of professors, coordinators and students from online undergraduate programs in Colombia regarding the use of communication tools, presentation and appropriation of contents. Despite some achievements, these findings highlight the need to emphasize a dialogic character interaction and collaboration within the spaces designed for this kind of communication. In addition, this paper highlights that information needs to be presented in different ways to motivate students to interact with the materials and to stimulate their understanding of contents.

\section{Transfer to practice}

Technological developments enable the pedagogical use of multiple tools to support the educational processes of an increasingly large number of students enrolled in online undergraduate programs. This research aims to encourage professors to move into the use of social networks, spaces of collective creation and creativity stimulation, in order to enhance the presentation and appropriation of program contents, and to generate processes of communication between students and professors in more meaningful and enlightening ways.

\section{Résumé}

Cet article de recherche présente les résultats d'une étude mixte qui a examiné les expériences et perceptions d'un groupe de professeurs, coordinateurs et étudiants de programmes de licence à modalité virtuelle en Colombie par rapport à l'emploie d'outils pour la communication, la présentation et l'appropriation de contenus. En dépit de quelques réussites, les trouvailles montrent le besoin de mettre l'accent sur l'interaction de caractère dialogique et la collaboration dans les espaces créés pour la communication. De la même façon, la représentation de l'information requière faire appel à d'autres formes différents au texte écrit qui puissent motiver aux étudiants à interagir avec les matériaux et stimulent leur compréhension des contenus.

\section{Transfert à la pratique}

Le progrès technologiques du réseau internet donne la possibilité d'un emploie pédagogique de multiples outils pour appuyer les processus éducatifs d'un nombre à chaque fois plus grand d'étudiants en programmes de licence à modalité virtuelle. Les résultats de cette recherche invitent à entrer dans l'usage des réseaux sociaux, espaces de création collective et espaces pour stimuler la créativité afin d'enrichir la présentation et appropriation de contenus des programmes et créer des processus de communication entre les étudiants et les professeurs plus significatifs et riches.

\section{Palavras chave}

Educação a distância; aprendizagem virtual; educação superior; comunicação; tecnologia da informação

\section{Resumo}

Este artigo de pesquisa expõe os resultados de um estudo misto que examinou as experiências e percepções de um grupo de professores, coordenadores e estudantes de programas de graduação na modalidade virtual na Colômbia a respeito do emprego de ferramentas para a comunicação, a apresentação e a apropriação de conteúdos. Apesar de alguns pontos positivos, o que se encontrou evidencia a necessidade de enfatizar a interação, em caráter dialógico, e a colaboração nos espaços desenhados para a comunicação. Da mesma forma a representação da informação requer recorrer a formas diferentes de texto escrito que motivem os estudantes a interagir com os materiais e estimulem seu entendimento de conteúdos.

\section{Transferência à prática}

Os avanços tecnológicos da web possibilitam o emprego pedagógico de múltiplas ferramentas para apoiar os processos educativos de um número cada vez maior de estudantes em programas de graduação na modalidade virtual. Os resultados desta pesquisa convidam a incursionar no uso de redes sociais, nos espaços de criação coletiva e nos espaços para o estímulo da criatividade em favor de uma apresentação mais rica e de uma maior apropriação dos conteúdos dos programas e assim gerar processos de comunicação mais significativos e enriquecedores entre estudantes e professores. 


\section{Introducción}

Las Tecnologías de la Información y la Comunicación (TIC) han servido como canal de expansión de la educación a distancia y virtual y han facilitado el acceso a un mayor número de estudiantes al sistema educativo. En Colombia, las políticas educativas del gobierno han permitido la puesta en marcha de programas en modalidad a distancia y virtual. Alrededor de 230 programas académicos de formación profesional en pregrado aparecen inscritos en las páginas oficiales del Ministerio de Educación Nacional con 18.447 estudiantes matriculados para el período 2012-2 (Ministerio de Educación Nacional, MEN, Sistema Nacional de Información de la Educación Superior, SNIES, 2016). Sin embargo, no se han realizado investigaciones sobre la implementación de ese tipo de programas y menos aún focalizadas en su valoración desde la perspectiva de estudiantes y profesores. Esta investigación representa una primera aproximación al examen de los avances, transformaciones y vacíos que se perciben en los programas que se ofertan.

El estudio recabó información para responder a la necesidad de documentar lo que acontece pedagógica y tecnológicamente en ese tipo de programas y proponer orientaciones para la mejora de los procesos de enseñanza y aprendizaje. El objetivo general fue explorar, a partir de las percepciones de estudiantes y docentes/tutores, la manera en que se está llevando a cabo la implementación del componente pedagógico y tecnológico en los programas de pregrado en modalidad virtual en Colombia. Uno de los objetivos específicos fue determinar los avances y obstáculos en el empleo de herramientas para la comunicación y la presentación de contenidos, ya que son dos ejes esenciales en la metodología que se emplea en la virtualidad. Aspectos relacionados con los modelos que se privilegian, los materiales, las metodologías y el desarrollo de la competencia informacional se reportan en otros trabajos (Pineda \& Díaz, 2012; Pineda \& Hennig, 2012; Pineda, Hennig \& Segovia, 2013; Pineda, Hennig, Segovia, Díaz, Sánchez, Otero \& Rees, 2012).

\section{Panorama sobre herramientas para la comunicación y para la presentación de contenidos}

Los desarrollos tecnológicos permiten un mayor empleo de formas variadas y creativas de representación de la información y del conocimiento (Ministerio de Educación Nacional, 2006). En la actualidad, se cuenta con una gama de alternativas que permiten que los estudiantes interactúen con diferentes formas de representación del conocimiento que posibilitan la abstracción de un objeto o situación de estudio. Este proyecto se basó en las formas de representación de contenidos y herramientas que ofrece la denominada Web 2.0., término acuñado en 2004 que alude al agregado tecnológico, que puesto al servicio de la educación, abarca una plétora de opciones interactivas, disponibles principalmente en internet y que según San Murugesan (2007), tiene como uno de sus objetivos la promoción del aprendizaje colaborativo y cooperativo.

Christine Greenhow, Beth Robelia y Joan E. Hughes (2009) añaden que la literatura que explora la Web 2.0, menciona reiteradamente que su empleo facilita la participación, la distribución de roles y estimula el uso de diferentes tipos de medios audiovisuales participativos. En general, es todo aquello que se pone a disposición del profesor y del estudiante para fomentar el intercambio de información, la interacción, el debate de ideas, la realización de tareas, bien sea por una comunicación escrita (correo
Descripción del artículo | Article description | Description de l'article | Artigo descrição

Este artículo de investigación hace parte del proyecto La configuración de las dimensiones pedagógica y tecnológica de los programas de educación virtual de pregrado de las IES en Colombia, realizado por los grupos Educación y Educadores, Facultad de Educación y Tecnologías para la Academia, y Proventus, Centro de Tecnologías para la Academia, financiado por la Universidad de La Sabana (EDU 28-2009) y cofinanciado por Colciencias (1230-489-25380) 
electrónico, chat, tablón de anuncios) o por una vía auditiva y audiovisual (audioconferencia y videoconferencia) y que propician, tanto una comunicación sincrónica como asincrónica (Cabero, 2006). Según Elena Barbera (2006), y Piia Näykki y Sanna Järvelä (2008), las formas de interacción que promuevan los programas de pregrado en modalidad virtual influirán en el fomento de la discusión argumentada para la construcción de conocimiento y en el impulso de un sistema cognitivo avanzado. Sin embargo, para que ello ocurra es indispensable el desarrollo de habilidades, por parte del profesor, para guiar a los participantes en la construcción de ideas con base en la perspectiva del otro y para estimular niveles más avanzados de pensamiento (Hou Chang \& Sung, 2009; Lucas, Gunawardena \& Moreira, 2014). Además, requiere que el profesor o facilitador monitoree de forma permanente los espacios de discusión virtual en aras de disminuir la elaboración de ideas o conceptos errados que se puedan generar en las discusiones (Yap \& Chia, 2010).

\section{Las posibilidades que ofrece la Web 2.0}

Algunos denominan los aplicativos de la Web 2.0 'software social' por el hecho de que promueven procesos reflexivos individuales pero sobre todo porque fomentan la interacción y el aprendizaje colaborativo (Kesim \& Agaoglu, 2007; Laru, Näykki \& Järvelä, 2012). Otros lo llaman web de la escritura y la lectura, porque efectivamente se producen y se consumen gran cantidad de textos (Hemmi, Bayne $\&$ Land, 2009) y reiteradamente, la literatura los califica como "dinámicos, interactivos, democráticos, centrados en la gente, volátiles, sociales y adaptativos" (Brown, 2012, p. 50). Estas características se incluyen en herramientas como weblogs, blogs, podcasts, internet forums, wikispaces, social networks, entre muchas otras. Los trabajos más recientes de Juan Manuel Trujillo-Torres, Francisco Javier Hinojo-Lucena e Inmaculada Aznar-Díaz (2011) y Juan Manuel Trujillo-Torres y Francisco Raso-Sánchez (2010) recalcan el valor de la Web. 2.0 en la evolución de los procesos de enseñanza y aprendizaje e-learning en la universidad. Los autores coinciden en señalar que su uso favorece la generación de un mayor sentido de compromiso por parte del estudiante con su proceso y que potencia el trabajo colaborativo, el diálogo y el intercambio efectivo de ideas en red. Así mismo, favorece el empleo de la experiencia docente de manera más contextualizada y significativa.

\section{Las redes sociales}

Facebook, Twitter, Hi5 y Flickr, por mencionar algunos, son espacios de socialización colectiva que forman parte de la cotidianidad de los individuos modernos y que han permeado la actividad académica. El estudio de Marisol Gómez-Aguilar, Sergio Roses-Campos y Pedro Farias-Batlle (2012) evidencia que se pueden emplear con fines académicos para la resolución de dudas, la actualización de información sobre clases y trabajos y para el intercambio de conocimiento. Trabajos interventivos, como el de Paulette Stewart (2009), centrado en el análisis de textos en círculos literarios, señalan que son una experiencia social de aprendizaje matizada por la afectividad y la colaboración. Christine Greenhow (2011), Muhammad Kamarul Kabilan, Norlida Ahmad y Mohamad Jafre Zainol Abidin (2010), Anne West, Jane Lewis y Peter Currie (2009) añaden que estos espacios estimulan la motivación, el compromiso y fortalecen el tejido relacional de las comunidades de aprendizaje. 
Las interacciones que emergen en las redes tienen injerencia en las competencias comunicativas, cognitivas y sociales de los estudiantes, lo que a su vez impacta la inteligencia interpersonal y el pensamiento crítico (Lampe, Ellison \& Steinfield, 2008) y aumenta el sentido de responsabilidad, autonomía (Joinson, 2008), autoconfianza y autoestima (Bosch, 2009). Además, brindan oportunidades para adoptar e interpretar roles diferentes, lo cual permite a los estudiantes examinar múltiples perspectivas y desarrollar tolerancia y respeto hacia la diversidad. Sin embargo, su carácter incontrolado podría generar una sobrecarga de información (Greenhow, 2011) y, además, por ser considerados frecuentemente como espacios de diversión y esparcimiento podrían generar distracción (Yu, Tian, Vogel \& Chi-Wai, 2010). También se discute su aplicabilidad en el contexto educativo, como el caso que presentan Gabriela Grosseck, Ramona Bran y Laurentiu Tiru (2011), que en su estudio experimental demostraron que solo el $10 \%$ de los participantes en un curso ofrecido por Facebook se sintió parte de una comunidad.

\section{Espacios para la construcción colectiva}

Los blogs y wikis son tal vez los medios más utilizados para fomentar la interacción y el trabajo colaborativo. La primera herramienta se emplea casi como un diario en que se comparte información con otros. Entre sus ventajas, Manuel Lorenzo-Delgado, Juan Manuel Trujillo-Torres, Rocío Lorenzo-Martín y Eufrasio PérezNavío (2011) mencionan que facilitan la categorización de trabajos, el registro de notas, el establecimiento de enlaces permanentes, las reflexiones constructivas, la realización de lecturas, exposiciones, ilustraciones, el uso de estudios de caso, y que se pueden apoyar en recursos multimodales (p. 143). La segunda herramienta se propone como un espacio en la web para presentar y compartir contenidos; pero a diferencia de los blogs, permiten su modificación. Ulrike Cress y Joachim Kimmerle (2008) enfatizan el potencial de esta herramienta por varias razones. En primer lugar, es de fácil acceso, ya que estos espacios están disponibles en la web, o pueden ser implementados en intranets que no requieren la instalación de software especializado. En segunda instancia, fomentan procesos escriturales colaborativos de creación, edición y producción de textos en los que la revisión entre pares juega un papel preponderante (Hsu, 2007). Los wikis facilitan la internalización de información por medio del trabajo colectivo y, a su vez, reflejan procesos y representaciones cognitivas individuales. Como toda herramienta virtual tiene aspectos positivos como la posibilidad de modificar el contenido de los escritos de una página de manera rápida y la promoción de la interacción, la libre creación y la expresión entre quienes los construyen. Empero, presenta dificultades relacionadas con el vandalismo por cuanto los contenidos pueden ser eliminados o modificados sin control alguno (Palomo, Ruiz \& Sánchez, 2008).

Otros espacios de creación colectiva involucran herramientas como los foros de discusión que se constituyen en espacios asincrónicos en los que el estudiante comparte información y articula "sus ideas y opiniones desde distintas fuentes de discusión, promoviendo el aprendizaje a través de varias formas de interacción, distribuidas en espacios y tiempos diferentes" (Arango, 2004, p. 3). En los foros se estimula la posibilidad de participación y de contribución al logro de objetivos académicos. Sin embargo, presentan dificultades relacionadas con la cantidad y la calidad de las intervenciones. En cuanto al primer aspecto, demandan del profesor un proceso de seguimiento y realimentación puntual y precisa que en ocasiones se ve afectado de manera negativa por el gran número de estudiantes que participan en esos espacios. En cuanto a la calidad de las intervenciones, requieren orientaciones puntuales para que las interacciones revistan un carácter verdaderamente dialógico y para que se fomente la colaboración. Estos mismos obstáculos también están presentes en los chats que son espacios de construcción colectiva pero de carácter sincrónico.

\section{Herramientas para estimular la creatividad}

Así como hay herramientas para fortalecer la comunicación, también hay otras diseñadas principalmente para estimular la creatividad. Los blogs y los mundos virtuales se incluyen en esta categoría. Weichao Chen y Curtis J. Bonk (2008) definen el blog como una página en la cual las entradas están "visualizadas por orden cronológico reverso" (p. 43) que promueve habilidades de recuperación, evaluación, organización y análisis de información. Esta herramienta también estimula la interactividad entre los estudiantes y desempeña un papel valioso en procesos de autoevaluación y heteroevaluación (Hsu, 2007). A diferencia de los foros y los chats, los blogs muestran un registro claro y permanente del progreso del estudiante y facilitan el seguimiento al desarrollo de sus ideas.

Los mundos virtuales, por su parte, son entornos simulados tridimensionales interconectados por medio de las computadoras (Dickey, 2003). Un aspecto fundamental de esta herramienta es su sentido de presencia en el tiempo y el espacio que se crea cuando los avatares, que son representaciones de los usuarios, interactúan en un entorno 3D realista (Dreher, Reiners, Dreher \& Dreher, 2009). Esta característica es de gran relevancia en la experimentación 
del sentido de presencia para el estudiante que participa en programas de educación a distancia (Eschenbrenner, Nah \& Siau, 2008; Lu, 2010). De manera más precisa, Li-Fen Lilly Lu (2010) señala que los sentidos de autopresencia y copresencia, y la presencia de lugares generados por medio de la visualización de un avatar, aumentan la participación, motivación y capacidad de aprendizaje del estudiante comparado con otras herramientas. Los mundos virtuales parecen desempeñar un papel importante en los procesos de aprendizaje significativo que van más allá de la memorización. Una crítica a los mundos virtuales es que su diseño y la creación de escenarios y avatares es una tarea dispendiosa y onerosa, aunque en la actualidad es posible encontrar algunos gratuitos en la red.

Uno de ellos, tal vez el más popular, es Second Life que cuando ha sido empleado con propósitos educativos ha tenido injerencia en la disminución de la ansiedad y el incremento de la motivación (Wehner, Gump \& Downey, 2011), en el goce y la creatividad (Ferguson, 2011), en el aprendizaje experiencial (Jarmon, Traphagan, Mayrath \& Trivedi, 2009) y en la inclusión de la inmediatez, término empleado por Traci Anderson (2009) que alude a elementos prosódicos, kinésicos y proxémicos que inciden de forma positiva en la comunicación en el aula de clase. Según esta autora, los mundos virtuales como Second Life permiten la creación de estos elementos siempre y cuando el docente haya desarrollado suficientemente sus competencias para el empleo de la herramienta.

En general, se puede afirmar que el empleo de estas herramientas exige del docente familiarización, práctica continua y principalmente una actitud reflexiva y selectiva para lograr que se acoplen a sus necesidades pedagógicas y no se conviertan simplemente en elementos de distracción carentes de un propósito educativo.

\section{Escenario de la investigación}

Esta investigación fue de carácter exploratorio-descriptivo, transversal y mixto. Se considera exploratoria, porque no se cuenta con estudios previos en el contexto colombiano en particular y, por ende, es una primera aproximación al estudio de la pedagogía y la tecnología de los programas de formación de pregrado en modalidad virtual y a distancia que estaban en funcionamiento en 2011. En cuanto a su carácter mixto, se apeló a la aplicación de instrumentos cuantitativos y cualitativos para obtener una visión más precisa y complementaria. Elssy Bonilla-Castro y Penélope Rodríguez-Sehk (2005) señalan que los estudios mixtos incrementan la probabilidad de conocer un objeto o sujeto de estudio, a partir de las bondades de cada enfoque. Para nuestro caso, el cuestionario y las entrevistas permitieron complementar los datos y profundizar en ellos.

\section{Población y muestra}

Se empleó un muestreo no probabilístico intencional (McMillan \& Schumacher, 2010), es decir, se seleccionaron programas de formación de pregrado con un fuerte componente virtual en el período acotado. Con base en ese criterio se identificaron 18 universidades, con 51 programas, a las cuales se invitó a formar parte del estudio. De este grupo, solo nueve aceptaron participar de manera voluntaria y de ellas solo seis (cinco privadas y una pública) permitieron que sus estudiantes y docentes respondieran una encuesta online que las instituciones se encargaron de enviar a sus estudiantes. Las tres restantes adujeron razones administrativas y logísticas para declinar la solicitud de aplicación de la encuesta. 


\section{Instrumentos}

La recolección de información incluyó dos momentos. Para el primero, se empleó una encuesta online que se diseñó y aplicó a profesores y estudiantes de programas de pregrado en modalidad virtual y a distancia en las instituciones de educación superior seleccionadas. La encuesta se diseñó con base en el aplicativo de Google.docs y fue validada por dos pares expertos. La totalidad de la encuesta para profesores está disponible en el enlace http://goo.gl/ydLNp y consta de 65 ítems de selección múltiple. La correspondiente a los estudiantes se ubica en http://goo.gl/3J5sR y contiene 52 peguntas.

De la población estudiantil, 288 diligenciaron el cuestionario. Su edad promedio fue 31,80 años, con una desviación típica de 8,31 años. La edad mínima registrada fue 16 y la máxima 59 años, lo cual demuestra una alta variabilidad en este grupo. La gráfica 1 señala que 161 (56\%) fueron mujeres y 127 hombres (44\%). Del grupo de docentes/tutores, 44 (70\% hombres y $30 \%$ mujeres) respondieron la encuesta. Su edad promedio fue 43,16 años con una desviación típica de 10,21 años. De ellos, 56,8\% pertenecían a instituciones públicas y $43,2 \%$ a privadas.

Gráfica 1

Distribución de la población por sexo (\%)

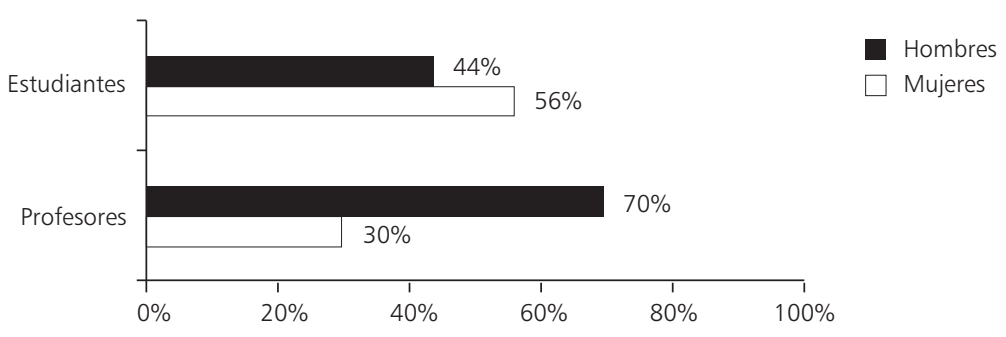

Fuente: elaboración propia

La gráfica 2 muestra que un alto porcentaje de estudiantes (43,4\%) pertenecía a programas del área administrativa y financiera, en menor proporción a carreras relacionadas con humanidades y contaduría $(20,1 \%$ y $18,8 \%$, respectivamente) y las menores agrupaciones dijeron pertenecer al área de ingeniería $(9,4 \%)$ y a campos de relaciones internacionales y políticas (8,3\%).

Gráfica 2

Distribución de los estudiantes por carreras (\%)

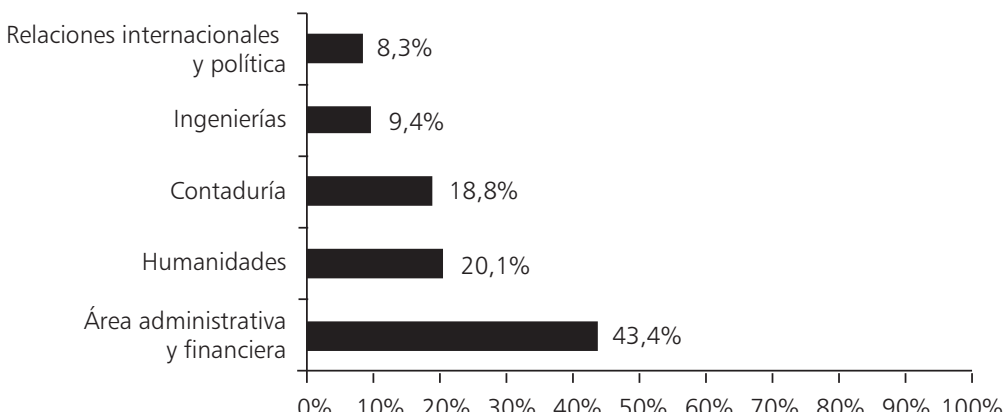

Fuente: elaboración propia 
El componente cualitativo implicó la realización de 48 entrevistas individuales y grupales con estudiantes y profesores que tuvieron una duración aproximada de 90 minutos. El contenido de las preguntas estuvo orientado a conocer más en detalle sobre: (1) los objetivos, contenidos, criterios de selección de contenidos, relación entre temas, conexiones de los temas con conocimientos previos de los estudiantes, procesos cognitivos que se privilegian, (2) las estrategias didácticas y los recursos que se emplean, las rutas para el fomento de la argumentación escrita y oral, (3) el fomento del trabajo colaborativo, el acompañamiento al estudiante, las herramientas para fomentar la interacción y el trabajo colaborativo, (4) cómo se estimula la investigación y se desarrolla la competencia informacional y (5) las formas de evaluación que más se emplean. En cuanto al componente ético, se siguieron los procedimientos establecidos para proyectos de investigación en ciencias sociales y se contó con el consentimiento informado de los sujetos. Su participación fue voluntaria y se garantizó el respeto a su dignidad e integridad.

\section{Análisis de datos}

Para el análisis descriptivo de las encuestas se empleó el paquete estadístico SPSS. La información se reportó con base en la frecuencia de respuestas y empleando porcentajes. Por su parte, las entrevistas fueron transcritas y se analizaron con base en los principios de la teoría fundamentada, sugeridos por Kathy C. Charmaz (2006). Su modelo incluye cinco fases en las que se identifican códigos, es decir, temas o conceptos que surgen de los datos mismos, se realizan agrupaciones de los temas que aparecen de manera recurrente y se clasifican en categorías a las que se les asigna un nombre. Luego se comparan y contrastan los datos y se proveen descripciones o explicaciones sobre las categorías y sus relaciones. Con base en este trabajo inductivo, se responden los objetivos de investigación. En reuniones periódicas, el grupo de investigación comparó y contrastó los análisis realizados de manera individual para asegurar su calidad, disipar dudas y ampliar el espectro de interpretación. La discusión de las interpretaciones con otros investigadores se empleó como una manera de asegurar la credibilidad y transparencia en el análisis y se conoce como triangulación por investigador (Merriam, 2009).

\section{Resultados}

A continuación, se describen los principales hallazgos de este trabajo que se agrupan en grandes categorías en las que se emplean datos que provienen de las entrevistas y de las encuestas. Los nombres seleccionados corresponden al tema principal que se identificó en las entrevistas y que se corroboró en las encuestas. Se describe en primera instancia las percepciones de los participantes acerca de las herramientas para la comunicación y más adelante, sus visiones sobre las herramientas para la presentación de contenidos en los cursos. Cada categoría se sustenta con datos puntuales de las encuestas y con extractos de las entrevistas, es decir, con las voces de los estudiantes y profesores/tutores que formaron parte del estudio.

\section{Correo electrónico y foro: sinónimos de comunicación en la virtualidad}

Los hallazgos de esta investigación revelan que el correo electrónico y los foros se sitúan como los principales mecanismos para establecer contacto entre tutores/docentes y sus estudiantes y entre estos últimos y que su empleo reviste un carácter informativo y de acompañamiento. Además de ofrecer datos puntuales para la entrega de trabajos, procesos académicos y administrativos, los docentes apelan al correo electrónico para guiar puntualmente a los alumnos en el desarrollo de las asignaturas y resolver dudas sobre los contenidos y ejercicios que se proponen. Los correos han generado lo que los profesores denominan una cultura de la comunicación. Es decir, son espacios compartidos por un grupo que mantiene intereses comunes centrados en la actualización e intercambio de información sobre los contenidos y tareas de las asignaturas. Los correos se asignan a los estudiantes al ingresar a los programas $y$, desde el punto de vista de los profesores, son espacios para el acompañamiento y fortalecimiento de procesos formales de escritura.

Lo que hoy trabajo lo hago dejándoles un plan de trabajo. Lo explico y lo envío por correo electrónico a cada estudiante. Se ha creado esa cultura en el estudiante (profesor, institución 1, p. 12).

Voy materia por materia, revisando el correo de la plataforma porque todos los días el profesor está mandando algo (estudiante, institución 1, p. 4).

Cuando [un estudiante] me escribe correos electrónicos, le corrijo la puntuación, de las palabras, todo (profesor, institución 2, p. 9).

Estas percepciones se corroboran en los datos suministrados en la encuesta. La gráfica 3 señala que tanto estudiantes como docentes utilizan mayoritariamente el correo electrónico ( $94 \%$ y $81 \%$, respectivamente), seguido por los foros (75\% docentes y $77 \%$ estudiantes) y en tercer lugar, el chat (67\% profesores y $57 \%$ estudiantes). Llama la atención el alto porcentaje de uso de llamadas telefónicas (46\% profesores y $41 \%$ estudiantes), lo cual muestra la necesidad 
percibida de contacto más personalizado y la baja frecuencia en el empleo de redes sociales y los video/audioconferencias.

Gráfica 3

Herramientas empleadas en los cursos para estimular la comunicación

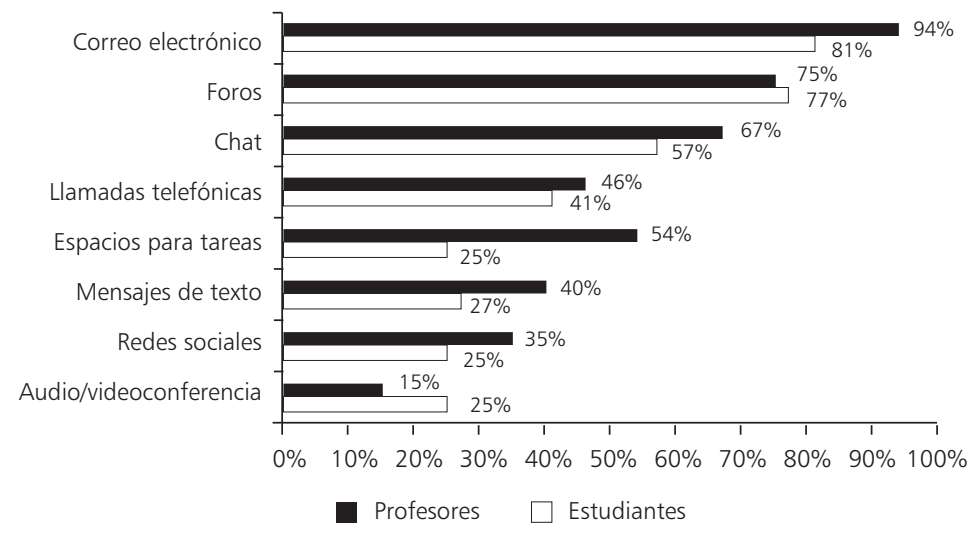

Fuente: elaboración propia

Los foros, aunque se emplean con bastante frecuencia, distan de ser espacios en los que se realiza una construcción colectiva de conocimiento. El trabajo colaborativo, aspecto central en esa construcción de conocimiento, aún tiende a concebirse como una simple distribución de tareas entre los estudiantes que conforman un equipo. En el ejemplo que se cita a continuación, el estudiante entrevistado refiere encuentros con sus compañeros para asignar tareas que se realizan de manera individual y luego se unifican en un solo producto que se sube al foro, con lo que se ilustra que los foros no se conciben como espacios para la interacción dialógica y el debate.

En este semestre tengo dos compañeros con los que hicimos como tres o cuatro trabajos... Los dividimos [los trabajos] y después lo ponemos todo junto y lo enviamos. O sea, antes de enviarlos, los unificamos (estudiante, institución 4, p. 3).

El trabajo colaborativo es uno de los grandes retos para los docentes de los programas virtuales, que están conscientes de que la participación y la realización de tareas colectivas se restringen a unos cuantos integrantes en los grupos. El producto que resulta de ese tipo de trabajo, desde el punto de vista de los profesores, es un aprendizaje fragmentado.

La verdad, la verdad es que implementar trabajo colaborativo en distancia y principalmente en el modelo que nosotros trabajamos es un poco complejo porque ¿cómo es el cuento? Uno trabaja, el otro lleva algo, y el otro se pega y pone el nombre (profesor 4, entrevista institución 2, p. 23).

Nosotros procuramos que los laboratorios sean hechos en grupo, pero a veces... Inicialmente ¿cuál fue la respuesta? Que tenemos respuestas fragmentadas. Entonces si eran 6 puntos, usted hace 3 y yo hago los otros 3 (profesor 1, entrevista institución 3, p. 12).

Las falencias en el trabajo colaborativo han suscitado reflexiones pedagógicas sobre el uso de las herramientas en mención y los docentes han 
comenzado a generar estrategias para controlar la participación de los estudiantes y para evidenciar que los trabajos sean el resultado de un trabajo colectivo. Tal es el caso de una institución que ha discutido el tema y que ha planeado apelar a otras herramientas para examinar cómo trabajan sus estudiantes:

Se ha pensado en unos protocolos para trabajo en grupo, en relatorías, en observación... Lo que se ha pensado de pronto es implementar unas de esas estrategias para efectivamente evidenciar que ellos están trabajando, que se reúnen a trabajar y no que una sola persona lo hace y los demás se pegan (profesor 3, entrevista institución 2, p. 24).

Por otra parte, se observó que, a pesar del adelanto tecnológico actual, persiste el empleo de medios tradicionales sincrónicos como la llamada telefónica. De manera recurrente se apela a ella para interactuar principalmente con los docentes, lo cual puede asociarse a que este medio permite más inmediatez en la respuesta. Sin embargo, como transformación en la comunicación sincrónica aparecen Skype y los chats, opciones que también facilitan la comunicación rápida e inmediata entre estudiante y profesor. A pesar de aparecer como dos alternativas para establecer contacto directo, su uso está restringido por las diferencias de horarios propias de las ocupaciones y procedencias de los participantes. El siguiente fragmento muestra la valoración del chat como herramienta que posibilita el contacto con el docente y en lo pedagógico permite la asesoría para aclaración de dudas sobre los temas tratados:

Eso también [chat] es una herramienta que ellos valoran mucho porque es la posibilidad de acceder a estar con uno. Claro que a veces uno hace chat extemporáneo porque ellos de pronto necesitan alguna asesoría en algo específico, o vieron un video y no lo entendieron o algo. Entonces hago chat extemporáneos y hay una parte del chat, que se llama Sala de Conversaciones, donde ellos pueden poner mensajes en horas extemporáneas y uno les contesta igual. O sea, no tiene que ser siempre a través del correo (profesora 2, entrevista institución 2 p. 17).

El empleo de herramientas como Skype o chats parece propiciar el acercamiento entre los agentes educativos e implícitamente lleva a pensar que ciertos rasgos de la presencialidad son aún indispensables en el proceso formativo de los estudiantes en modalidad virtual. De hecho, algunos docentes señalan que prevalecen los sentimientos de aislamiento y soledad y que herramientas como el teléfono o Skype ayudan a reducir esas sensaciones.

A veces, el estudiante sí experimenta esa sensación de soledad. Somos de esa cultura que necesita del abrazo, de compañía. Entonces hay mucha demanda, mucho reclamo de ese acompañamiento. Ahí tratamos de compensar el acompañamiento siendo muy afectivos, además de muy exigentes. Acompañarlo y decirle que ahí estamos, que finalmente la modalidad virtual hacia allá apunta (profesor, institución 3, p. 13).

Algunos estudiantes ratificaron la necesidad de emplear herramientas que permitieran un contacto más visual con sus docentes, porque al parecer influye en la manera en que procesan la información que reciben.

Quizá como tener un contacto más visual con el docente porque la parte visual es importante... ver el docente, escucharlo, verlo escribir... uno eso 
es lo que muchas veces le hace entender muchas cosas, [ver] el docente escribiendo en un tablero dándote clase (estudiante, entrevista institución 6, p. 10).

Las redes sociales, finalmente, son las herramientas de comunicación que se emplean con menor frecuencia (35\% estudiantes y $25 \%$ profesores) y de hecho, los estudiantes en las entrevistas señalaron que su empleo se circunscribe al campo de relaciones sociales. Muy pocas instituciones acuden a estos espacios como instrumento de comunicación y su uso se limita a la divulgación de eventos, noticias o publicidad institucional.

\section{La prevalencia de textos escritos 'tradicionales' para la presentación de contenidos}

En relación con los recursos empleados por los docentes para la presentación de los contenidos de sus cursos, la gráfica 4 muestra los resultados obtenidos. Tanto estudiantes como profesores señalan que los contenidos se presentan mayoritariamente en textos escritos en formatos Word y PDF (93\% profesores y $98 \%$ estudiantes). Un alto porcentaje también alude al empleo de videos, gráficos e imágenes fijas.

Gráfica 4

Herramientas para la presentación de contenidos

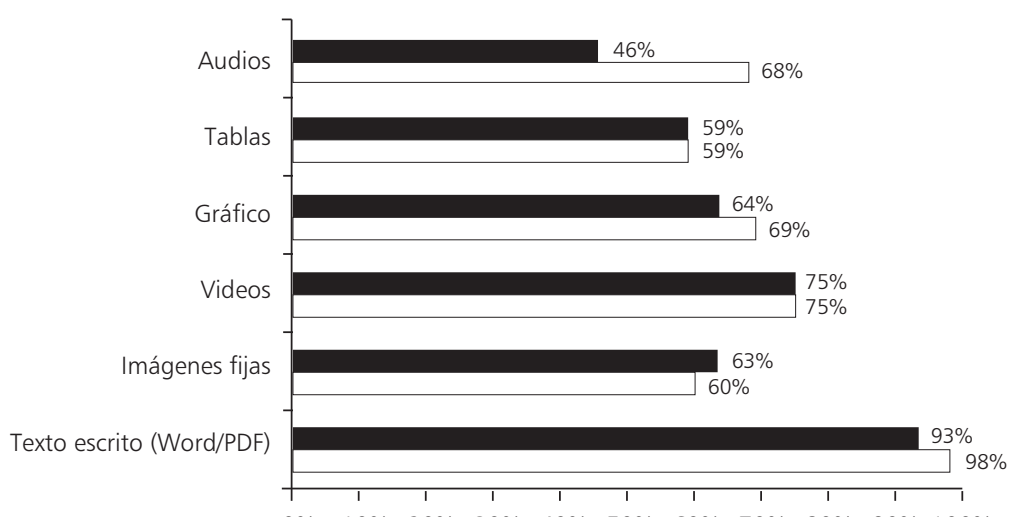

$\begin{array}{llllllllllll}0 \% & 10 \% & 20 \% & 30 \% & 40 \% & 50 \% & 60 \% & 70 \% & 80 \% & 90 \% & 100 \%\end{array}$

Profesores $\square$ Estudiantes

Fuente: elaboración propia

Las declaraciones de docentes y estudiantes en las entrevistas guardan coherencia con los resultados de las encuestas. La presentación de contenidos se hace con base en materiales en formato Word y PDF, lo cual implica que no se están empleando otros recursos que podrían resultar igualmente útiles o incluso más motivantes. Los profesores emplean enlaces para la búsqueda de otros recursos, pero parece que la competencia informacional de los estudiantes no está suficientemente estimulada y desarrollada pues ellos los perciben como un "material adicional":

Vienen [los recursos para la presentación de contenidos] en la plataforma, como tal, en formato PDF. Todos vienen así. Los enlaces son como adicionales. Como si dijeran: busque aquí y encontrará el enlace en el que puede buscar tal cosa. Pero la mayoría vienen en formato PDF (estudiante, institución 4, p. 2). 
Es más como hacia el texto, escritos y hacia formatos. Yo también les doy material complementario, que tengo desarrollado para esos temas y que están en Power Point. Se los mando el primer día de clases. Les mando el material el primer día de clases para que lo puedan usar (profesor, institución 1, p. 5)

Sin embargo, varias de las instituciones que formaron parte de este proyecto cuentan con unidades especializadas en el diseño, implementación y evaluación de recursos para la presentación de contenidos y su rasgo característico es el trabajo colaborativo entre profesores, diseñadores, asesores pedagógicos y técnicos. Es notoria la producción de videos como una alternativa para enriquecer la experiencia de los estudiantes.

\footnotetext{
Nosotros tenemos videos, teleconferencias y todo eso lo realizamos nosotros... Aquí hay unos presentadores que ya están seleccionados. El guion literario que es el documento en el que se basa una videoconferencia o la audioconferencia, lo realiza el docente o el director de programa (coordinadora, institución 5, p. 17).
}

El empleo de videos constituye el pilar para la exploración de temáticas con un alto componente práctico o de aplicación y para casos en los que se desea imprimir mayor dinamismo y variedad a la práctica. Un programa en particular cuenta con una galería virtual en la que los estudiantes encuentran videos para modelos de fisioterapia:

Hay una galería virtual, que tiene una tutoría virtual como por ejemplo para fisioterapia, cómo hacer calentamiento previo a un deporte. Lo podemos tener totalmente visual en esta plataforma (coordinadora, institución 5, p. 22).

No obstante, algunos estudiantes mencionaron su dificultad para el estudio y principalmente para la realización de tareas que demandan el empleo de símbolos y fórmulas en operaciones matemáticas. La ausencia de software especializado incide en la realización de actividades en tiempos prudentes con mayor concentración y profundidad. Esta carencia duplica el tiempo de realización de tareas pues los estudiantes se ven obligados a elaborar sus respuestas en papel, escanear sus trabajos y luego remitirlos por correo a los tutores.

Al principio fue muy complicado. Nos tocaba hacerla a mano porque la matemática toca hacerla a mano primero para luego pasarla. Entonces era un complique. Escaneábamos los procesos para que el profesor viera lo que nosotros hicimos y escribíamos la respuesta porque no nos alcanzaba el tiempo para más. De todas maneras no existe un programa que sea automático y coloque todo en campos que pueda llenarse (estudiante, institución 2, p. 8).

\section{Los grandes ausentes: herramientas para la creatividad y los espacios de creación colectiva}

Si bien los programas indagados muestran avances en el empleo de diversas herramientas para poner en marcha sus planes de estudio, se observó que los wikis, blogs, las redes sociales, los mundos virtuales y los juegos no aparecen como formas de apoyo en la educación virtual de las instituciones de educación superior, IES, colombianas, con excepción de 
un par de casos aislados en los que los profesores dicen emplear blogs y Second Life. Esta omisión conduce a reflexionar sobre la apropiación de los docentes de las herramientas que ofrece la Web 2.0.

En la gráfica 5 se aprecian los resultados sobre el uso de wikis y blogs por parte de estudiantes y profesores.

Gráfica 5

Uso de wikis y blogs

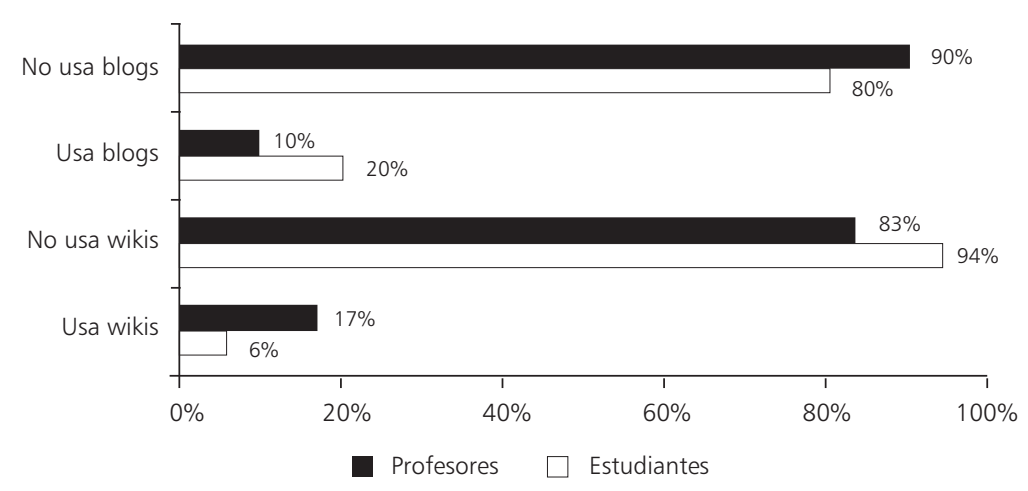

Fuente: elaboración propia

Se observa una baja frecuencia de uso de estas herramientas para los dos grupos encuestados. Los wikis son usados por el 6\% de los estudiantes y el $17 \%$ de los profesores, mientras que los blogs por el $10 \%$ y $20 \%$, respectivamente. Los datos de las entrevistas confirman el bajo uso de esas herramientas por cuanto al preguntar acerca de su aplicación en los programas, los estudiantes mencionaron que no se empleaban. Solo en algunos casos aislados, los docentes aludieron al empleo de blogs, a su intención de generar interacción, pero se plantean como intentos tímidos para la dinamización de contenidos:

El blog tiene que ser una herramienta que me permita interactuar con el estudiante. Yo me tomé el atrevimiento de proponer entre las temáticas, el uso del blog, pero combinarla con algunas herramientas de la Web 2.0, y empezamos a incorporar videos, PDF, incorporar presentaciones al blog, para que mejoren la dinámica del contenido (coordinador, institución 8, p. 18).

\section{Conclusiones y discusión}

El objetivo del trabajo que se expone en este documento fue presentar un panorama sobre los avances y dificultades en el uso de herramientas para la comunicación y presentación de contenidos en los programas de formación de pregrado en modalidad virtual en Colombia. En general, los resultados muestran algunos avances, pero algunas herramientas son subutilizadas en este contexto y hay un largo camino que recorrer para incentivar a los profesores en su empleo. A continuación, se presentan las conclusiones y se discuten a la luz de otros trabajos científicos sobre el tema.

Si bien los hallazgos de esta investigación evidencian que los foros y los correos electrónicos se emplean con frecuencia para estimular la comunicación entre estudiantes y profesores, también suscitan grandes dudas sobre su aplicación para favorecer la colaboración y la construcción de conocimiento. El énfasis en los aportes individuales y aislados sin un intercambio y discusión de ideas podría ser un indicador de que estos recursos 
carecen de un matiz dialógico. En este sentido, valdría la pena hacer un análisis del papel que cumplen los profesores como moderadores u orientadores de las interacciones con esas herramientas. El presente estudio, al igual que las investigaciones mencionadas en la introducción, considera esencial que el docente actúe como catalizador de la interacción que se propicia en las herramientas para la comunicación.

Seguir estimulando el empleo de los foros constituye una pieza fundamental para un trabajo constructivo y colaborativo en la educación virtual. Pedro A. Willging (2008) asegura que el uso de foros asíncronos para enseñanza y aprendizaje en línea ha sido ampliamente adoptado por las instituciones que ofrecen programas y cursos en la modalidad a distancia. Mantener a los estudiantes comprometidos en esas discusiones en línea es vital por tres razones. En primer, lugar se estimula el aprendizaje colaborativo; en segunda instancia, se incrementa el diálogo constructivo y por último, se proveen oportunidades para la socialización. Sin embargo, es necesario considerar que a diferencia de lo que ocurre en las clases presenciales, donde los gestos y las señales visuales y/o verbales le pueden dar al instructor una idea de la motivación y el nivel de interés de sus estudiantes, en el foro de discusión asíncrono (generalmente, basado en texto), no es fácil evaluar el nivel de motivación y compromiso de los participantes.

Lo anterior conduce a reflexionar sobre la idea de que la educación virtual demanda claridad y precisión sobre lo que realmente implica el trabajo colaborativo. Incuestionablemente, este campo requiere fortalecimiento en la educación virtual colombiana. Romper el imaginario de que el trabajo en grupo se puede equiparar con el trabajo colaborativo es tal vez el primer paso para lograr un trabajo mancomunado entre docentes y estudiantes y entre estos últimos, para el logro de un aprendizaje significativo. En este sentido, los programas de capacitación y profesionalización docente deben propender por el fomento del análisis de conceptos claves en los modelos pedagógicos que sustentan la educación virtual. Diferenciar el trabajo colaborativo del trabajo en grupo es, por consiguiente, imprescindible para el logro de un aprendizaje activo y significativo en esta modalidad. La comprensión sobre lo que implica el trabajo colaborativo llevará a estimular adecuadamente la interacción entre pares y a estructurar actividades de aprendizaje de manera intencional para que los estudiantes se comprometan a trabajar en colectivos que cumplan los objetivos de aprendizaje propuestos.

De otra parte, la llamada telefónica se sigue empleando como una de las principales herramientas de comunicación y los chats y Skype surgen como alternativas que ofrecen rasgos de presencialidad que parecen tener gran importancia en el proceso formativo de los estudiantes en esta modalidad, por cuanto ayudan a confrontar sentimientos de aislamiento y soledad. Este hallazgo conduce a pensar también en que el acompañamiento del profesor debe estar matizado por un sentido más humano de la comunicación. En su reflexión sobre las relaciones valorativas y afectivas en la educación virtual, Héctor Eduardo Cardona-Carmona (2008) propone que las estructuras cognitivas no deben excluir lo concerniente a la dimensión humana y, por ende, "la emoción no puede ser excluida de la enseñanza y del aprendizaje, fragmentando al ser humano en partes racionales e irracionales" (p. 8). Esta afirmación adquiere gran sentido y relevancia en esta investigación. Si bien los programas en modalidad virtual emplean el chat, e-mail y los foros para promover la comunicación y la interacción, es preponderante rescatar el valor de un intercambio que apunte a un acercamiento más cálido y que ayude a combatir el sentimiento de aislamiento y soledad que aún perciben los estudiantes en esta modalidad educativa. En este sentido, un derrotero es el empleo de Skype o de plataformas como Elluminate que incluyen espacios sincrónicos de interacción en los que la presencialidad y la corporeidad juegan un papel importante. En concordancia con lo anterior, Leah P. Macfadyen (2011) invita a reflexionar sobre las implicaciones sociales, culturales, experienciales y cognitivas de la corporeidad en el aprendizaje y argumenta que los indicadores corporales de identidad como gestos, acento y entonación son elementos ausentes en las formas tradicionales de comunicación en la educación virtual. La inclusión de estos elementos ofrece un matiz de proximidad y afectividad que sin duda influye de manera positiva en la dimensión humana del proceso educativo en la virtualidad. Los estudios realizados por Yeferson Romaña-Correa (2015), Mario Guerrero (2014) y Aida Cuestas-Verjano (2013) señalan, además, las virtudes del empleo de Skype como herramienta pedagógica en el aprendizaje y como facilitadora del proceso de interacción y recalcan su valor al generar autenticidad en las actividades que se proponen para los estudiantes.

Una alternativa que ofrece un campo amplio de exploración para abrir canales de comunicación más afectivos son las redes sociales. Por consiguiente, se hace un llamado a la capacitación y/o actualización docente en aras de conocer o profundizar sobre su riqueza pedagógica. Si bien la literatura internacional evidencia su valor cuando se emplean de manera intencional con actividades claras y pertinentes, en Colombia es posible que aún no se empleen con propósitos netamente educativos o que no se esté haciendo un seguimiento y documentación de experiencias sobre la aplicación de estas iniciativas. 
Esta investigación evidencia que aún hay un largo camino por recorrer en el uso de herramientas para la representación de contenidos pues los objetos de aprendizaje y las actividades están asociadas y, de alguna manera, restringidas a textos escritos principalmente en formatos Word y PDF. Persiste el uso de material impreso, tendencia que coincide con otros trabajos y que deja entrever "la urgente necesidad de clarificar lo que comprende una metodología a distancia y/o virtual, para garantizar una enseñanza de calidad" (Ruiz-Corbella, García-Aretio, Álvarez-González \& Rubio-Gómez, 2011, p. 239).

Este resultado muestra una inminente necesidad de aventurarse a la exploración de múltiples herramientas virtuales con propósitos pedagógicos. Las instituciones de educación superior con programas de formación de pregrado en modalidad virtual requieren un uso más categórico de las posibilidades tecnológicas que ofrece la Web 2.0 y que incluyen un sinnúmero de herramientas digitales para compartir información, interactuar y colaborar en la construcción de conocimientos. Esta propuesta, sin embargo, nos lleva a reflexionar sobre dos aspectos claves. Uno se asocia al sistema de apoyo con que cuentan las instituciones para cualificar a los docentes de educación virtual en la producción de materiales que contengan un matiz multimodal y que propendan por la interacción dialógica y el trabajo colaborativo. Si bien algunas instituciones cuentan con equipos especializados para el diseño de sus recursos, se requiere reforzar el trabajo entre los equipos técnicos y los docentes para garantizar que la información se represente en formas multimodales diversas y para que se dinamicen las opciones de intercambio de saberes. Un segundo aspecto se condensa en las preguntas: ¿quién es el profesor que participa en la educación virtual colombiana y cuáles son sus necesidades de formación y profesionalización en este campo? Se podría especular que muchos docentes migran de la educación tradicional a la virtual sin un conocimiento o profundización sobre lo que implica el campo de la virtualidad y, por consiguiente, sus opciones pedagógicas se circunscriben a la transferencia de las prácticas que se realizan en los programas presenciales.

Este trabajo también exhorta a la comunidad docente en programas virtuales y a distancia a incursionar en el empleo de mundos virtuales, sin obviar que su uso debe someterse a juicio crítico y al análisis permanente sobre los resultados que produce en relación con la construcción de conocimiento. Si bien sus ventajas se describen en la introducción de este documento, es pertinente mencionar el trabajo de Konstantinidis Andreas, Thrasyvoulos Tsiatsos, Theodouli Terzidou y Andreas Pomportsis (2010), que incluye evidencias sobre actitudes negativas por parte de los estudiantes en el empleo de esa herramienta, principalmente cuando se compara con otras como foros, videoconferencias y correos electrónicos. Esta visión negativa se exacerba si se carece de experiencia en navegación y familiarización con juegos de computador en versión 3D (Jarmon, Traphagan, Mayrath \& Trivedi, 2009) y si no se cuenta con una estructura tecnológica apropiada. En este sentido, vale la pena examinar si las plataformas tecnológicas con las que cuentan las instituciones colombianas tienen la capacidad para el sustento de este tipo de propuestas.

En relación con lo anterior, es indispensable considerar que lo mismo ocurre con otras posibilidades como los juegos educativos, principalmente aquellos disponibles en línea. Ciertamente, este campo ha sido poco explorado en nuestro contexto. Los juegos representan oportunidades para el dominio de contenidos, estimulan la aventura, incrementan la motivación, la interacción y la comunicación, el dominio de conocimiento factual, procedimental, estratégico, metacognitivo y fomentan el empleo del 
pensamiento crítico y la resolución de problemas (Annetta, Folta \& Klesath, 2010).

Es deseable por consiguiente, hacer un Ilamamiento a los programas en modalidad virtual para aprovechar las ventajas que ofrecen las novedosas herramientas disponibles en la Web 2.0 e incluirlas en sus propuestas pedagógicas. Estimular su empleo se fundamenta en varias razones. Una es que en la actualidad muchos estudiantes llegan a las aulas con experiencias en herramientas tecnológicas que incluyen diversos elementos visuales, hipertexto y movimiento, que hacen que sus cerebros estén moldeados para absorber rápidamente información presentada de formas variopintas. Otra es que muchos de ellos desarrollan tareas simultáneas -o multitareas-, con diferentes recursos tecnológicos, lo cual estimula todos sus sentidos y facilita la adaptación para la realización de diferentes tipos de actividades (Annetta, Folta \& Klesath, 2010). Una tercera razón está asociada con el incremento en la motivación, la interacción, la formación de comunidades de aprendizaje que consecuentemente redunda en "la construcción y gestión del conocimiento desde una estructura nodal interconexionada" (Trujillo-Torres, Hinojo-Lucena \& Aznar-Díaz, 2011, p. 144).

Sin embargo, es imperativo no dejar al margen tres aspectos fundamentales. Uno involucra que cualquiera que sea la herramienta o la actividad que se utilice debe siempre contar con un trasfondo educativo en el que se priorice el logro de objetivos. El segundo es que la capacidad reflexiva y crítica de diseñadores, expertos temáticos y sobre todo de los profesores, debe prevalecer para tomar decisiones informadas sobre la aplicabilidad y utilidad de esas herramientas y actividades. En este sentido, también es imperativo asegurar que los agentes educativos no se dejen 'seducir' por la novedad y el goce exclusivamente, sino que prioricen el acompañamiento y andamiaje en el proceso de aprendizaje, ideas que han sido sugeridas en otros trabajos (Azevedo \& Jacobson, 2008). El tercer aspecto se asocia al tipo de estudiante que ingresa a los programas virtuales en Colombia. Si bien la tendencia mundial es al ingreso de un mayor número de "nativos digitales", también se tendría que examinar cuidadosamente si nuestros estudiantes cumplen ese perfil o, de lo contrario, estaríamos enfrentándolos a desafíos que podrían desestimularlos.

\section{Limitaciones}

Por último es importante mencionar algunas limitaciones del presente estudio que inciden en su alcance. En primera instancia, la reducida participación de las instituciones convocadas y en segundo lugar que, a pesar de que algunas de ellas facilitaron la realización de las entrevistas a directivos, docentes y encargados de los aspectos tecnológicos y pedagógicos de cada uno de los programas, no permitieron la aplicación de las encuestas a los estudiantes. Una posible línea de actuación para convocar a las instituciones a revisar sus propuestas en aras de mejorar su calidad podría ser la creación de redes o conglomerados que faciliten mecanismos de seguimiento y evaluación y que se nutran de sus propias experiencias en este campo. De igual manera, esta investigación sugiere continuar profundizando en el estudio del campo virtual en Colombia focalizado por ejemplo en aspectos como la interacción, o en el estudio de propuestas ejemplares que puedan emplearse como modelos a seguir.

\section{Sobre los autores}

María Patricia Otero de Suárez es magíster en educación, Universidad de La Sabana. Fisioterapeuta, especialista en docencia universitaria y terapia manual. Directora del programa de fisioterapia, Universidad de La Sabana. Miembro de los grupos de investigación Educación y Educadores y Movimiento Corporal Humano, Universidad de La Sabana.

Clelia Pineda-Báez es PhD en educación (currículo e instrucción). Magíster en TEFL, Southern Illinois University, Carbondale, Estados Unidos. Docente investigadora de la maestría en educación, Facultad de Educación, Universidad de La Sabana. Miembro de los grupos de investigación Educación y Educadores, Universidad de La Sabana.

Geraint Paul Rees es doctorando en traducción y ciencias del lenguaje. Máster en Lingüística Teórica e Aplicada, Universitat Pompeu Fabra, Barcelona. Magíster en Educación, Universidad de La Sabana. Docente investigador de la Universitat Pompeu Fabra. Becario predoctoral del Institut Universitari de Lingüística Aplicada (IULA). Miembro del grupo de investigación InfoLex.

\section{Referencias}

Anderson, T. (2009). Online Instructor Immediacy and Instructor-Student Relationships in Second Life. En Charles Wankel \& Jan Kingsley (eds.). Higher Education in Virtual Worlds: Teaching and Learning in Second Life, 101-115. United Kingdom: Emerald Group Publishing Limited.

Andreas, K.; Tsiatsos, T.; Terzidou, T. \& Pomportsis, A. (2010). Fostering Collaborative Learning in Second Life: Metaphors and Affordances. Computers \& Education, 55, 603-615. Disponible en: http:// www.researchgate.net/publication/223180919_ Fostering_collaborative_learning_in_Second_ Life_Metaphors_and_affordances

Annetta, L.; Folta, E. \& Klesath, M. (2010). V-Learning: Distance Education in the $21^{\text {st }}$ Century through $3 D$ Virtual Learning Environments. New York: Springer. 
Arango, M. L. (2004). Foros virtuales como estrategia de aprendizaje. Revista Debates Latinoamericanos, 2 (1), 1-21.

Azevedo, R. \& Jacobson, M. (2008). Advances in Scaffolding Learning with Hypertext and Hypermedia: A Summary and Critical Analysis. Education Technology Research Development, 56 (1), 93-100.

Barbera, E. (2006). Collaborative Knowledge Construction in Highly Structured Virtual Discussions. The Quarterly Review of Distance Education, 7 (1), 1-12.

Bonilla-Castro, E. \& Rodríguez-Sehk, P. (2005). Más allá del dilema de los métodos: la investigación en ciencias sociales. Bogotá: Grupo Editorial Norma.

Bosch, T. E. (2009). Using Online Social Networking for Teaching and Learning: Facebook Use at the University of Cape Town. Communicatio: South African Journal for Communication Theory and Research, 35 (2), 185-200. DOI: 10.1080/02500160903250648

Brown, S. (2012). Seeing Web 2.0 in Context: A Study of Academic Perceptions. Internet and Higher Education, 15 (1), 50-57.

Cabero, J. (2006). Bases pedagógicas del E-learning. Revista Universidady Sociedad del Conocimiento, 3 (1), 1-10. Disponible en: http://www. uoc.edu/rusc/3/1/dt/esp/cabero.pdf

Cardona-Carmona, H. E. (2008). Consideraciones acerca de la educación virtual como comunidad de relaciones afectivo-valorativas. Revista Iberoamericana de Educación, RIE, 46 (7), 7-25. Disponible en: http:// www.rieoei.org/deloslectores/2203Carmona.pdf

Charmaz, K. C. (2006). Constructing Grounded Theory: A Practical Guide through Qualitative Analysis. Thousand Oaks, California: Sage.

Chen, W. \& Bonk, C. (2008). The Use of Weblogs in Learning and Assessment in Chinese Higher Education: Possibilities and Potential Problems. International Journal on E-Learning, 7 (1), 41-65.

Cress, U. \& Kimmerle, J. (2008). A Systemic and Cognitive View on Collaborative Knowledge Building with Wikis. Computer-Supported Collaborative Learning, 3 (2), 105-122. Disponible en: http://www. researchgate.net/publication/220309175_A_systemic_and_ cognitive_view_on_collaborative_knowledge_building_with_Wikis

Cuestas-Verjano, A. (2013). Using Skype in a Primary Class. A Case Study. Bellaterra Journal of Teaching \& Learning Language \& Literature, 6 (2), 49-68. Disponible en: http://ddd.uab.cat/pub/jtl3/jtl3_a2013m56v6n2/jtl3_a2013m5-6v6n2p49.pdf

Dickey, M. D. (2003). Teaching in 3D: Pedagogical Affordances and Constraints of 3D Virtual Worlds for Synchronous Distance Learning. Distance Education, 24 (1), 105-121. Disponible en: https://www. academia.edu/7848907/Teaching_in_3D_Pedagogical_Affordances_ and_Constraints_of_3D_Virtual_Worlds_for_Synchronous_Distance_ Learning

Dreher, C.; Reiners, T.; Dreher, H. \& Dreher, N. (2009). Virtual Worlds as a Context Suited for Information Systems Education: Discussion of Pedagogical Experience and Curriculum Design with Reference to Second Life. Journal of Information Systems Education, 20 (2), 211-224. Disponible en: http://www.researchgate.net/publication/230854701_ Virtual_Worlds_as_a_Context_Suited_for_Information_Systems Education_Discussion_of_Pedagogical_Experience_and_Curriculum_ Design_With_Reference_to_Second_Life

Eschenbrenner, B.; Nah, F. F.- H. \& Siau, K. (2008). 3-D Virtual Worlds in Education: Applications, Benefits, Issues, and Opportunities. Journal of Database Management, 19 (4), 91-110. 
Ferguson, R. (2011). Meaningful Learning and Creativity in Virtual Worlds. Thinking Skills and Creativity, 6 (3), 169-178.

Gómez-Aguilar, M.; Roses-Campos, S. \& Farias-Batlle, P. (2012). El uso académico de las redes sociales en universitarios. Comunicar. Revista Científica de Comunicación y Educación, 38 (19), 131-138. Disponible en: http://www.revistacomunicar.com/index.php?contenido=detalle s\&numero=38\&articulo $=38-2012-16$

Greenhow, C. (2011). Online Social Networks and Learning. On the Horizon, 19 (1), 4-12. DOI 10.1108/10748121111107663

Greenhow, C.; Robelia, B. \& Hughes, J. (2009). Learning, Teaching, and Scholarship in a Digital Age. Web 2.0 and Classroom Research: What Path Should We Take Now? Educational Researcher, 38 (4), 246-259.

Grosseck, G.; Bran, R. \& Tiru, L. (2011). Dear Teacher, What Should I Write on my Wall? A Case Study on Academic Uses of Facebook. Procedia - Social and Behavioral Sciences, 15 (0), 1425-1430. DOI: 10.1016/j. sbspro.2011.03.306

Guerrero, M. (2012). The Use of Skype as a Synchronous Communication Tool between Foreign Language College Students and Native Speakers [El uso de Skype como herramienta de comunicación sincrónica entre estudiantes universitarios y hablantes nativos]. HOW, 19, 3347. Disponible en: howjournalcolombia.org/index.php/how/article/ download/36/36

Hemmi, A.; Bayne, S. \& Land, R. (2009). The Appropriation and Repurposing of Social Technologies in Higher Education. Journal of Computer Assisted Learning, 25 (1), 19-30. Disponible en: http://www.sianbayne. net/pdfs/jcal_paper.pdf

Hou, H. T.; Chang, K. E. \& Sung, Y. T. (2009). Using Blogs as a Professional Development Tool for Teachers: Analysis of Interaction Behavioral Patterns. Interactive Learning Environments, 17 (4), 325-340.

Hsu, J. (2007). Innovative Technologies for Education and Learning: Education and Knowledge-Oriented Applications for Blogs, Wikis, Podcasts and More. International Journal of Information and Communication Technology Education, 3 (3), 70-89.

Jarmon, L.; Traphagan, T.; Mayrath, M. \& Trivedi, A. (2009). Virtual World Teaching, Experiential Learning and Assessment: An Interdisciplinary Communication Course in Second Life. Computers and Education, 53 (1), 169-182.

Joinson, A. N. (2008). 'Looking at', 'Looking up' or 'Keeping up with' People? Motives and Uses of Facebook. Proceedings of the $26^{\text {th }}$ Annual SIGCHI [Special Interest Group on Computer-Human Interaction] Conference on Human Factors in Computing Systems Florence, Italy, April 05-10 2008, 1027-1036. New York: Association for Computing Machinery, ACM. Disponible en: http://onemvweb.com/sources/sources/looking_ at_motives_facebook.pdf

Kabilan, M. K.; Ahmad, N. \& Abidin, M. J. Z. (2010). Facebook: An Online Environment for Learning of English in Institutions of Higher Education? Internet and Higher Education, 13 (4), 179-187.

Kesim, E. \& Agaoglu, E. (2007). A Paradigm Shift in Distance Education: Web 2.0 and Social Software. Turkish Online Journal of Distance Education, 8 (3), 66-75. Disponible en: https://www.academia. edu/4944377/A_Paradigm_Shift_in_Distance_Education_Web_2.0_ and_Social_Software

Lampe, C.; Ellison, N. \& Steinfield, C. (2008). Changes in Use and Perception of Facebook. Proceedings of the ACM 2008 Conference on Computer Supported Cooperative Work, 721-730. New York: 
Association for Computing Machinery, ACM. Disponible en: https://www.msu.edu/ nellison/ LampeEllisonSteinfield2008.pdf

Laru, L.; Näykki, P. \& Järvelä, S. (2012). Supporting Small-Group Learning Using Multiple Web 2.0 Tools: A Case Study in the Higher Education Context. Internet and Higher Education, 15 (1), 29-38.

Lorenzo, M.; Trujillo, J.; Lorenzo, R. \& Pérez, E. (2011). Usos del weblog en la universidad para gestión de conocimiento y trabajo en red. Pixel-Bit: Medios y Educación, 39, 141-154. http://acdc. sav.us.es/pixelbit/images/stories/p39/11.pdf

Lu, L. (2010). Teaching $21^{\text {st }}$ Century Art Education in a Virtual Age: Art Café @ Second Life. Art Education, 63 (6), 19-24.

Lucas, M.; Gunawardena, C. \& Moreira. A. (2014). Assessing Social Construction of Knowledge Online: A Critique of the Interaction Analysis Model. Computers in Human Behavior, 30, 574-582.

Macfadyen, L. P. (2011). Being and Learning in the Online Classroom: Linguistic Practices and Ritual Text Acts. En Robin Goodfellow \& Mary-Noëlle Lamy (eds.). Learning Cultures in Online Education, 93-112. Lexington, Kentucky: Continuum.

McMillan, J. \& Schumacher, S. (2010). Research in Education. Evidence-Based Inquiry. Boston: Pearson.

Merriam, S. B. (2009). Qualitative Research: A Guide to Design and Implementation. San Francisco, California: John Wiley\& Sons.

Ministerio de Educación Nacional, MEN (2006). Visión 2019: tres programas estratégicos para mejorar la calidad y la competitividad de las personas y del país. Incorporación de las Tecnologías de la Información y la Comunicación (TIC) a los procesos educativos. Disponible en: http://www. mineducacion.gov.co/1621/articles-101945 archivo_pdf1.pdf

Ministerio de Educación Nacional, MEN, Sistema Nacional de Información de la Educación Superior, SNIES (2016). http://bi.mineducacion. gov.co:8380/eportal/web/snies1/metodologiadel-programa

Murugesan, S. (2007). Understanding Web 2.0. IT Professional, 9, 34-41. Disponible en: http:// www.researchgate.net/publication/3426898_ Understanding_Web 2.0

Näykki, P. \& Järvelä, S. (2008). How Pictorial Knowledge Representations Mediate Collaborative Knowledge Construction in Groups. Journal of Research on Technology in Education, JRTE, 40 (3), 359-387. Disponible en: http://files.eric. ed.gov/fulltext/EJ826082.pdf

Palomo, R.; Ruiz, J. \& Sánchez, J. (2008). Enseñanza con TIC en el siglo XXI. La escuela 2.0. Sevilla: Editorial Mad.
Pineda, C. \& Díaz, D. (2012). La construcción del material educativo en modalidad virtual: ventajas del ejercicio participativo. Congreso Internacional Docencia Universitaria e Innovación, CIDUI, La Universidad: una Institución de la Sociedad, Barcelona, España: 4-6 julio de 2012. Disponible en: http://www.cidui.org/revista-cidui12/index. php/cidui/article/view/288/281

Pineda, C. \& Hennig, C. (2012). Estudios de caso y ABP en programas de pregrado en modalidad virtual: rutas para estimular la indagación y la autonomía: Problematización, indagación y soluciones vinculadas a la realidad laboral del estudiante de la modalidad virtual. Congreso Internacional Docencia Universitaria e Innovación, CIDUI, La Universidad: una Institución de la Sociedad, Barcelona, España: 4-6 julio de 2012. Disponible en: http://www.cidui.org/revista-cidui12/index. php/cidui/article/view/19/12

Pineda, C.; Hennig, C. \& Segovia, Y. (2013). Modelos pedagógicos, trabajo colaborativo e interacción en programas virtuales de pregrado en Colombia: un camino por recorrer. Revista de Universidad y Sociedad del Conocimiento, RUSC, 10 (2), 187-202. Disponible en: http://dialnet.unirioja. es/servlet/articulo?codigo $=4635330$

Pineda, C.; Hennig, C.; Segovia, Y.; Díaz, D.; Sánchez, M.; Otero, P. \& Rees, P. (2012). Alfabetización informacional en la educación superior virtual: logros y desafíos. Información, Cultura y Sociedad, 26, 83-104. Disponible en: http://eprints.rclis. org/19622/, http://eprints.rclis.org/19622/1/ n26a05.pdf

Romaña-Correa, Y. (2015). Skype ${ }^{\text {TM }}$ Conference Calls: A Way to Promote Speaking Skills in the Teaching and Learning of English. Profile Issues in Teachers' Professional Development, 17 (1), 143-156. Disponible en: http://www.revistas.unal.edu.co/ index.php/profile/article/view/41856/49766

Ruiz-Corbella, M.; García-Aretio, L.; Álvarez-González, B. \& Rubio-Gómez, M. (2011). Movilidad virtual en másteres a distancia en Europa y América Latina. Un camino por recorrer. Revista Iberoamericana de Educación, 55, 225-242. Disponible en: http://www.researchgate.net/ publication/235664937_Movilidad_virtual_ en_msteres_a_distancia_en_Europa_y_ Amrica_Latina._Un_camino_por_recorrer, http://rieoei.org/rie55a10.htm

Stewart, P. (2009). Facebook and Virtual Literature Circle Partnership. Building a Community of Readers. Knowledge Quest/Social Scholarship, 37 (4), 28-33.

Trujillo-Torres, J. M.; Hinojo-Lucena, F. J. \& Aznar-Díaz, I. (2011). Propuestas de trabajo innovadoras 
y colaborativas e-learning 2.0 como demanda de la sociedad del conocimiento. Estudios sobre Educación, ESE, 20, 141-159. Disponible en: http://dadun.unav.edu/bitstream/10171/18343/2/ ESE\%20141-159.pdf

Trujillo-Torres, J. M. \& Raso-Sánchez, F. (2010). Formación inicial docente y competencia digital en la convergencia europea (EEES) [Initial Teacher Training and Digital Competences in the European Convergence Process]. Enseñanza \& Teaching, Revista Interuniversitaria de Didáctica, 28 (1), 49-77. Disponible en: http://campus.usal.es/ revistas_trabajo/ index.php/0212-5374/article/view/7481/7946

Wehner, A. K.; Gump, A. W. \& Downey, S. (2011). The Effects of Second Life on the Motivation of Undergraduate Students Learning a Foreign Language. Computer and Assisted Language Learning, 24 (3), 277-289.

West, A.; Lewis, J. \& Currie, P. (2009). Students' Facebook 'Friends': Public and Private Spheres. Journal of Youth Studies, 12 (6), 615-627. Disponible en: http://www.researchgate.net/publication/249006058 Students Facebook friends Public and private spheres

Willging, P. (2008). Técnicas para el análisis y visualización de interacciones en ambientes virtuales. Revista Hispana para el Análisis de Redes Sociales, REDES, 14 (6), 1-23. Disponible en: http://revista-redes. rediris.es http://revistes.uab.cat/redes/article/view/v14-willging/133

Yap, K. C. \& Chia, K. P. (2010). Knowledge Construction and misconstruction: A Case Study Approach in Asynchronous Discussion Using Knowledge Construction - Message Map (KCMM) and Knowledge Construction - Message Graph (KCMG). Computers \& Education, 55 (4), 1589-1613. Disponible en: https://www.academia.edu/4564641/ Knowledge_construction_and_misconstruction_A_case_study_ approach_in_asynchronous_discussion_using_Knowledge_ Construction_-_Message_Map_KCMM_and_Knowledge Construction_-_Message_Graph_KCMG

Yu, A. Y.; Tian, S. W.; Vogel, D. \& Kwok, R. C.-W. (2010). Can Learning Be Virtually Boosted? An Investigation of Online Social Networking Impacts. Computers \& Education, 55 (4), 1494-1503. Disponible en: http://uhl2332gaga.wikispaces.com/file/view/SNW.pdf 